\title{
Intact and C-Terminal FGF23 Assays-Do Kidney Function, Inflammation, and Low Iron Influence Relationships With Outcomes?
}

Citation for published version (APA):

Sharma, S., Katz, R., Bullen, A. L., Chaves, P. H. M., de Leeuw, P. W., Kroon, A. A., Houben, A. J. H. M., Shlipak, M. G., \& Ix, J. H. (2020). Intact and C-Terminal FGF23 Assays-Do Kidney Function, Inflammation, and Low Iron Influence Relationships With Outcomes? Journal of Clinical Endocrinology \& Metabolism, 105(12), [665]. https://doi.org/10.1210/clinem/dgaa665

Document status and date:

Published: 01/12/2020

DOI:

10.1210/clinem/dgaa665

Document Version:

Publisher's PDF, also known as Version of record

Document license:

Taverne

Please check the document version of this publication:

- A submitted manuscript is the version of the article upon submission and before peer-review. There can be important differences between the submitted version and the official published version of record.

People interested in the research are advised to contact the author for the final version of the publication, or visit the DOI to the publisher's website.

- The final author version and the galley proof are versions of the publication after peer review.

- The final published version features the final layout of the paper including the volume, issue and page numbers.

Link to publication

\footnotetext{
General rights rights.

- You may freely distribute the URL identifying the publication in the public portal. please follow below link for the End User Agreement:

www.umlib.nl/taverne-license

Take down policy

If you believe that this document breaches copyright please contact us at:

repository@maastrichtuniversity.nl

providing details and we will investigate your claim.
}

Copyright and moral rights for the publications made accessible in the public portal are retained by the authors and/or other copyright owners and it is a condition of accessing publications that users recognise and abide by the legal requirements associated with these

- Users may download and print one copy of any publication from the public portal for the purpose of private study or research.

- You may not further distribute the material or use it for any profit-making activity or commercial gain

If the publication is distributed under the terms of Article 25fa of the Dutch Copyright Act, indicated by the "Taverne" license above, 


\title{
Intact and C-Terminal FGF23 Assays-Do Kidney Function, Inflammation, and Low Iron Influence Relationships With Outcomes?
}

\author{
Shilpa Sharma, ${ }^{1,2}$ Ronit Katz, ${ }^{3}$ Alexander L. Bullen, ${ }^{4}$ Paulo H.M. Chaves, ${ }^{5}$ \\ Peter W. de Leeuw, $^{6}$ Abraham A. Kroon, ${ }^{6}$ Alfons J.H.M. Houben, ${ }^{6}$ \\ Michael G. Shlipak, ${ }^{7}$ and Joachim H. Ix ${ }^{4,8}$
}

'Division of Nephrology, David Geffen School of Medicine at University of California Los Angeles, Los Angeles, California 90095; ${ }^{2}$ Nephrology Section, Veteran Affairs Greater Los Angeles Healthcare System, Los Angeles, California 90073; ${ }^{3}$ University of Washington, Seattle, Washington 98195; ${ }^{4}$ Division of NephrologyHypertension, Department of Medicine, University of California San Diego, San Diego, California 92103; ${ }^{5}$ Benjamin Leon Center for Geriatric Research and Education, Department of Translational Medicine, Herbert Wertheim College of Medicine, Florida International University, Miami, Florida 33199; ${ }^{6}$ Department of Internal Medicine and CARIM School for Cardiovascular Diseases, Maastricht University Medical Centre, 6200 MD Maastricht, the Netherlands; ${ }^{7}$ Department of Medicine, University of California San Francisco 94143; and ${ }^{8}$ Nephrology Section, Veterans Affairs San Diego Healthcare System, La Jolla, California 92161

ORCiD number: 0000-0001-9011-0163 (S. Sharma).

Abbreviations: ACR, albumin to creatinine ratio; CHS, Cardiovascular Health Study; CKD, chronic kidney disease; CRP, C-reactive protein; CVD, cardiovascular disease; EDTA, ethylenediaminetetraacetic acid; eGFR, estimated glomerular filtration rate; FGF23, fibroblast growth factor-23; HF, heart failure; MI, myocardial infarction; UACR, urine albumin to creatinine ratio.

Received: 16 July 2020; Accepted: 18 September 2020; First Published Online: 19 September 2020; Corrected and Typeset: 19 October 2020.

\section{Abstract}

Context: Higher fibroblast growth factor-23 (FGF23) concentrations are associated with heart failure and mortality in diverse populations, but the strengths of associations differ markedly depending up on which assay is used.

Objective: We sought to evaluate whether iron deficiency, inflammation, or kidney function account for differences in the strengths of associations between these 2 FGF23 assays with clinical outcomes.

Design: Case cohort study from the Cardiovascular Health Study.

Setting: A total of 844 community-dwelling individuals aged 65 years or older with and without chronic kidney disease were followed for 10 years.

Outcomes: Outcomes included death, incident heart failure (HF), and incident myocardial infarction (MI). Exposure was baseline intact and C-terminal FGF23. Using modified Cox models, adjusting sequentially we tested whether observed associations of each assay with outcomes were attenuated by iron status, inflammation, kidney function, or their combinations. Results: FGF23 measured by either assay was associated with mortality in unadjusted analysis (intact FGF23 hazard ratio [HR] per 2-fold higher 1.45; 95\% Cl, 1.25-1.68; C-terminal 
FGF23 HR 1.38; 95\% Cl, 1.26-1.50). Adjustment for kidney function completely attenuated associations of intact FGF23 with mortality (HR 1.00;95\% Cl, 0.85-1.17), but had much less influence on the association of C-terminal FGF23, for which results remained significant after adjustment (HR 1.15; 95\% Cl, 1.04-1.28). Attenuation was much less with adjustment for iron status or inflammation. Results were similar for the HF end point. Neither C-terminal or intact FGF23 was associated with MI risk.

Conclusions: The relationship of FGF23 with clinical end points is markedly different depending on the type of FGF23 assay used. The associations of biologically active FGF23 with clinical end points may be confounded by kidney disease, and thus much weaker than previously thought.

Freeform/Key Words: FGF23, assays, mortality, inflammation, kidney function, iron deficiency

Fibroblast growth factor-23 (FGF23) is a hormone produced by osteocytes in bone that influences the kidney to induce greater urinary phosphate excretion and to inhibit calcitriol production. In individuals with chronic kidney disease (CKD), FGF23 concentrations are markedly elevated, likely in an effort to avert hyperphosphatemia in the setting of diminished glomerular filtration rate. In animal models, FGF23 directly induces left ventricular hypertrophy (1), and prior epidemiological studies have linked higher concentrations of FGF23 with risk of heart failure (HF) and mortality. Relationships are also evident with atherosclerotic events such as myocardial infarction (MI) and stroke, but comparatively weaker $(2,3)$. FGF23 has been proposed as a potential causal intermediary between CKD and the HF and mortality end points. This has fueled interest in FGF23-lowering therapies, several of which are now the focus of phase 2 clinical trials $(4,5)$. The design of these trials, and subsequent phase 3 outcome trials, hinge heavily on the strength of association of FGF23 with clinically relevant end points, which is in question based on the assays used for FGF23 measurement.

There are 2 main types of assays for measuring FGF23 in humans. The C-terminal assay recognizes 2 epitopes on the C-terminus aspect of the hormone, capturing both intact FGF23 and its C-terminal fragments when the active hormone is cleaved and inactivated (6). Intact assays use antibodies that capture epitopes on either side of the FGF23 cleavage site, thus they capture only intact, biologically active FGF23 (7). The majority of prior studies reporting associations of FGF23 with mortality, HF, and cardiovascular disease (CVD) events have used C-terminal FGF23 assays (2). The few studies that have used intact FGF23 assays have found much weaker or altogether absent associations with the same end points $(8,9)$, and 2 recent systematic reviews both demonstrated marked heterogeneity in the strengths of associations with outcomes based on the FGF23 assay used for measurement in the underlying individual studies $(10,11)$.
Recent clinical and preclinical studies have demonstrated that iron deficiency and inflammation induce both FGF23 production and its cleavage, thereby increasing blood concentrations of C-terminal fragments without significantly influencing concentrations of the intact, biologically active FGF23 hormone. Inflammation and iron deficiency are much more common in patients with a greater burden of illnesses, and are themselves known to be associated with mortality risk $(12,13)$. Moreover, previous studies have demonstrated that intact FGF23 may be cleared from the body by the kidneys (14), and the relative abundance of C-terminal vs intact FGF23 varies by the severity of kidney function in humans (15). Factors such as kidney function, iron deficiency, and inflammation may have confounded the strong associations of C-terminal FGF23 with clinical outcomes reported in prior studies. Thus, the true strength of the associations of intact, biologically active, FGF23 hormone with clinical end points remains uncertain. If indeed weaker than with C-terminal assays, this would be an important obstacle for designing clinical trials testing FGF23-modifying therapies because sample size and statistical power estimates are markedly influenced by the strength of these associations.

The aforementioned systematic reviews have compared strengths of associations by assay type comparing one group of underlying studies vs another. However, whether kidney function, inflammation, and iron deficiency are potential confounders and account for the differences in strengths of association between the 2 types of FGF23 assays within the same individuals is uncertain. In the Cardiovascular Health Study (CHS) study, we previously reported strong associations of C-terminal FGF23 concentrations with $\mathrm{HF}$ and mortality, and weaker but existing associations with CVD events (2). At the time of our prior publication, neither intact FGF23 measurements nor iron deficiency markers were available in the CHS. In the present study, we sought to directly compare the strength of association of the 2 assays with 
clinical end points in the same individuals. In addition, we wanted to investigate the confounding influence of kidney function, inflammation, and iron deficiency on the associations of each assay with clinical end points. Thus, we used stored specimens from the CHS to add intact FGF23 measurements, in addition to iron and inflammatory biomarkers. A priori, we hypothesized that the association of C-terminal FGF23 with risk of mortality, HF, and MI would be stronger than with intact FGF23, and that adjusting models for iron deficiency and inflammation would attenuate association of C-terminal FGF23 resulting in associations more similar to those with intact FGF23.

\section{Materials and Methods}

\section{Study participants and setting}

The CHS design, objectives, sampling strategies, and examination techniques have been described previously (2). Briefly, eligibility required age 65 years or older, expectation to remain in the area for 3 years after recruitment, and no active cancer treatment. Between 1989 and 1990, a total of 5201 participants were recruited from 4 US communities using Medicare eligibility lists. An additional 687 individuals predominantly of African American race were recruited in 1992 and 1993. In-person examinations were performed annually through 1998 and 1999, and again in 2005 and 2006. Telephone interviews were conducted semiannually from 1989 to 1999 and biannually thereafter. We previously made C-terminal FGF23 measurements at the 1996 to 1997 study visit, selected because it was the first visit at which urine albumin to creatinine ratio (UACR) was measured and available within the cohort (2).

Participants were followed for mortality, HF, and CVD end points that were centrally adjudicated against prespecified criteria by the CHS Events Committee. Follow-up and adjudication continued until 2017. The study achieved $100 \%$ complete follow-up for ascertainment of mortality status.

\section{Study design and measurements}

We used a case-cohort design for this study. The casecohort design has advantages in that a single subcohort comparison group can be used for multiple outcomes (such as incident MI and HF herein), the subcohort is representative of the entire study population, and unbiased estimates of both absolute risks and relative hazards can be obtained without measurement of FGF23 in the entire cohort.

Among the 3107 individuals who participated and provided blood at the 1996 to 1097 visit, we randomly selected a sample of the cohort $(\mathrm{N}=844)$ for this analysis. We identified subsequent cases of incident HF $(\mathrm{N}=487)$ and incident MI $(\mathrm{N}=162)$. Per usual case-cohort design, cases could overlap with the random subcohorts by chance. Owing to the age and length of follow-up in the CHS, there were ample mortality events available within the 844-person subcohort, eliminating the need to identify mortality cases occurring outside the subcohort. We evaluated the mortality end point using a standard prospective cohort design and Cox proportional hazards models for the mortality end point.

\section{Fibroblast growth factor-23 measurements}

We had previously measured C-terminal FGF23 using fasting (8-hour) EDTA specimens collected at the 1996 to 1997 study visit that were stored at $-70^{\circ} \mathrm{C}$ until 2010 , using a C-terminal enzyme-linked immunosorbent assay (ELISA) kit (Immutopics) as described previously (2). In the present study, we measured intact FGF23 in previously unthawed EDTA plasma using the Kainos ELISA (Kainos Laboratories). Measurements were made in duplicate and averaged. Our estimates of the intra-assay and interassay coefficients of variation (CVs) were less than $5.0 \%$ to $8.7 \%$.

Information on baseline confounders was obtained at the 1996 to 1997 study visit concurrent with FGF23 measurements as described previously (2). Baseline confounders included age, sex, race, self-reported health status, and CVD risk factors including hypertension (systolic blood pressure $\geq 140 \mathrm{~mm} \mathrm{Hg}$, diastolic blood pressure $\geq 90 \mathrm{~mm} \mathrm{Hg}$, or use of antihypertensive medications), impaired fasting glucose (fasting glucose 100 to $125 \mathrm{mg} / \mathrm{dL}$ ), diabetes (fasting glucose $\geq 126 \mathrm{mg} / \mathrm{dL}$ or use of antiglycemic medications or insulin), smoking (current, former, or never), body mass index, total cholesterol, calcium, phosphate, parathyroid hormone, $25-\mathrm{OH}$ vitamin $\mathrm{D}$, use of lipid-lowering medications, and C-reactive protein (CRP) concentrations. Cystatin C concentrations were measured using a $\mathrm{BN}$ II nephelometer (Siemens) and subsequently estimated glomerular filtration rate (eGFR) was calculated using the CKD-EPI (Chronic Kidney Disease Epidemiology Collaboration) equation (16). A random morning urine sample was obtained and measured for urine albumin by rate nephelometry and creatinine using a Kodak Ektachem 700 Analyzer, and UACR was calculated $(\mathrm{mg} / \mathrm{g})$. Ferritin concentrations were measured in EDTA plasma using a Beckman Coulter clinical analyzer. The assay uses a latex agglutination reaction with spectrophotometric measurement. The analytic range is 8 to $450 \mathrm{ng} / \mathrm{mL}$ and intra-assay and interassay CVs are less than $5 \%$. Iron and total iron binding capacity were measured using serum specimens and the Beckman Coulter clinical analyzer. The analytic range for total iron binding capacity 
is 10 to $1000 \mu \mathrm{g} / \mathrm{mL}$ and intra-assay and interassay CVs are less than $6 \%$.

\section{Statistical analyses}

We categorized participants into quartiles based on the distribution of intact and C-terminal FGF23 concentrations in the random subcohort, and evaluated the distribution of confounders across FGF23 quartiles. Next, we examined the unadjusted correlations between the 2 FGF23 assays with one another, and with transferrin saturation, ferritin, CRP, eGFR, and UACR using Spearman correlation coefficients.

Sequential models were developed for each end point using identical series of models. Model 1 was unadjusted. Model 2 adjusted for ferritin and transferrin saturation only. Model 3 adjusted for CRP only. Model 4 adjusted for eGFR and UACR only. Finally, model 5 simultaneously adjusted for ferritin, transferrin saturation, CRP, eGFR, and UACR. These models allowed us to isolate the confounding influence of each of the variables of interest. Finally, we evaluated models that also adjusted for demographics and traditional CVD risk factors.

For the incident $\mathrm{HF}$ and incident MI end points, we used modified Cox regression to account for the case-cohort approach using inverse probability weights for the sampling probabilities $(17,18)$. These analyses included all 844 participants within the random subcohort and all incident $\mathrm{HF}$ and MI cases, regardless of whether they arose from the subcohort or the additional case sampling. Subcohort participants with prevalent HF and MI were excluded at baseline. Subcohort participants (HF/MI noncases and subcohort cases before the occurrence of event) were weighted by the inverse of the sampling fraction. Cases that arose outside the subcohort were not weighted before their failure. All cases (irrespective of whether they arose in the subcohort) were assigned a weight of 1 at the time of failure. Robust variance estimators were computed. Finally, we also assessed for effect modification by baseline CKD status (eGFR $<60 \mathrm{~mL} / \mathrm{min} / 1.73 \mathrm{~m}^{2}$ vs greater) for each outcome. All analyses were performed using R Core Team (2019) (R Foundation for Statistical Computing) and SPSS statistical software (IBM SPSS Statistics for Windows, version 26.0: IBM Corp 2019). A 2-sided $P$ value of less than .05 was considered statistically significant for all analyses including interaction terms.

\section{Results}

Among the 844 participants in the subcohort, the mean age was $78 \pm 5$ years, $62 \%$ were women, mean eGFR was $63 \pm 13 \mathrm{~mL} / \mathrm{min} / 1.73 \mathrm{~m}^{2}$, and median UACR was $8.6 \mathrm{mg} / \mathrm{g}$ (interquartile range [IQR], 4.8-19.5 mg/g). Median intact and C-terminal FGF23 concentrations were $61 \mathrm{pg} / \mathrm{mL}$ (IQR, 48-78 pg/mL) and 69 relative units (RU)/mL (IQR 53-95 RU/ $\mathrm{mL}$ ), respectively. Table 1 depicts the distribution of demographics, kidney function, and CVD risk factors across intact FGF23 quartiles. Similar data evaluating distribution of these variables across quartiles of C-terminal FGF23 are shown in Table 2. With either assay type, participants in higher quartiles of FGF23 had a greater burden of diabetes, hypertension, CKD, higher levels of CRP, and parathyroid hormone concentrations compared to those in the lowest quartile.

\section{Associations of iron status, inflammation, and chronic kidney disease indices with fibroblast growth factor-23 by assay type}

Within the random subcohort, we found the correlation of the 2 FGF23 assays with one another was of moderate strength $(r=0.48)$. Higher concentrations of C-terminal FGF23 were correlated with lower ferritin $(r=-0.26)$, lower transferrin saturation $(r=-0.28)$, and higher CRP $(r=0.21)$ concentrations. In contrast, higher concentrations of intact FGF23 were not significantly correlated with ferritin $(r=0.04)$ or transferrin saturation $(r=-0.03)$, and were only weakly correlated with CRP $(r=0.08$; Table 3$)$. We found similar correlations of C-terminal FGF23 and intact FGF23 with eGFR ( $r=-0.45$ and -0.42 , respectively) and UACR ( $r=0.19$ and 0.12 , respectively).

\section{Associations of intact and C-terminal fibroblast growth factor-23 with clinical outcomes}

In unadjusted analyses, higher FGF23 by either assay type was associated with mortality and incident HF. For both outcomes, associations were stronger with the C-terminal relative to the intact FGF23 assay. FGF23 concentrations were not associated with incident MI, irrespective of assay type (Table 4). Simple adjustment for ferritin and transferrin saturation had little influence on the association of intact FGF23 with mortality, but appeared to strengthen the association of C-terminal FGF23 with mortality (see Table 4). Adjustment for CRP concentrations in isolation had little influence on the association of either intact or C-terminal FGF23 with mortality. In contrast, simple adjustment for eGFR and UACR had marked effects on associations of FGF23 with mortality, but this differed by assay type. Adjustment for eGFR and ACR completely extinguished the association of intact FGF23 with mortality risk, taking the hazard ratio (per 2-fold higher) of intact FGF23 from 1.45 to 1.00. Adjustment for eGFR and ACR also attenuated the association of C-terminal FGF23 with mortality risk, but the degree of attenuation was much more modest (from 1.38 to 1.15), and in contrast to intact FGF23, the association of 
Table 1. Baseline characteristics by quartiles of intact fibroblast growth factor-23 in community-living older adults

\begin{tabular}{|c|c|c|c|c|c|}
\hline Variable & Full subcohort & Quartile 1 & Quartile 2 & Quartile 3 & Quartile 4 \\
\hline Intact FGF23 range, $\mathrm{pg} / \mathrm{mL}$ & & $<48$ & $48-61$ & $62-78$ & $>78$ \\
\hline No. & 844 & 210 & 213 & 208 & 213 \\
\hline Age, y & $78(5)$ & $78(5)$ & $78(5)$ & $78(4)$ & $79(5)$ \\
\hline Female & $519(62)$ & $136(64)$ & $137(64)$ & $127(61)$ & $119(56)$ \\
\hline Black & $122(15)$ & $44(21)$ & $28(13)$ & $31(15)$ & $19(9)$ \\
\hline$<$ High school education & $131(16)$ & $29(14)$ & $28(13)$ & $36(18)$ & $38(18)$ \\
\hline \multicolumn{6}{|l|}{ Smoking status } \\
\hline Never & $422(51)$ & $106(52)$ & $107(51)$ & $105(52)$ & $104(49)$ \\
\hline Former & $349(42)$ & $82(40)$ & $87(41)$ & $83(41)$ & $97(46)$ \\
\hline Current & $57(7)$ & $16(8)$ & $18(9)$ & $13(7)$ & $10(5)$ \\
\hline Body mass index, $\mathrm{kg} / \mathrm{m}^{2}$ & $27.0(4.8)$ & $26.4(4.8)$ & $26.5(4.6)$ & $27.4(4.8)$ & $27.8(4.9)$ \\
\hline Diabetes & $116(14)$ & $27(13)$ & $27(13)$ & $29(14)$ & $33(16)$ \\
\hline Hypertension & $500(60)$ & $118(56)$ & $126(59)$ & $111(54)$ & $145(68)$ \\
\hline Systolic blood pressure, $\mathrm{mm} \mathrm{Hg}$ & $137(21)$ & $138(23)$ & $137(21)$ & $134(19)$ & $138(21)$ \\
\hline Diastolic blood pressure, $\mathrm{mm} \mathrm{Hg}$ & $69(11)$ & $71(11)$ & $69(12)$ & $70(9)$ & $68(12)$ \\
\hline Heart rate, per min & $63(11)$ & $64(10)$ & $63(12)$ & $63(11)$ & $62(10)$ \\
\hline Antihypertensive med. use & $470(56)$ & $105(50)$ & $110(52)$ & $106(51)$ & $149(70)$ \\
\hline Total cholesterol, mg/dL & $202(39)$ & $200(41)$ & $203(40)$ & $201(35)$ & $202(40)$ \\
\hline Statin use & $96(11)$ & $18(9)$ & $27(13)$ & $18(9)$ & $33(16)$ \\
\hline \multicolumn{6}{|l|}{ Laboratory indices } \\
\hline $\mathrm{eGFR}, \mathrm{mL} / \mathrm{min} / 1.73 \mathrm{~m}^{2}$ & $63(18)$ & $73(17)$ & $66(17)$ & $63(16)$ & $52(18)$ \\
\hline $\mathrm{eGFR}<60$ & $354(42)$ & $49(23)$ & $77(36)$ & $89(43)$ & $139(65)$ \\
\hline Urine albumin/creatinine, $\mathrm{mg} / \mathrm{g}$ & $8.60(4.83-19.49)$ & $7.43(4.70-16.22)$ & $8.36(4.66-16.35)$ & $8.54(4.85-18.02)$ & $10.87(5.23-26.47)$ \\
\hline UACR $\geq 30 \mathrm{mg} / \mathrm{g}$ & $148(18)$ & $28(13)$ & $32(15)$ & $39(19)$ & $49(23)$ \\
\hline $25(\mathrm{OH})$ vitamin $\mathrm{D}, \mathrm{ng} / \mathrm{mL}$ & $28(11)$ & $26(11)$ & $28(11)$ & $29(11)$ & $30(11)$ \\
\hline Ferritin, ng/mL & $101(57-176)$ & $93(47-166)$ & $99(62-176)$ & $99(59-188)$ & $108(61-176)$ \\
\hline Transferrin saturation, $\%$ & $30(10)$ & $31(10)$ & $30(9)$ & $30(10)$ & $30(10)$ \\
\hline C-reactive protein, $\mathrm{mg} / \mathrm{L}$ & $2.34(1.04-5.04)$ & $2.05(0.94-4.61)$ & $2.46(1.15-5.43)$ & $2.24(0.97-4.43)$ & $2.78(1.35-5.83)$ \\
\hline Calcium, mg/dL & $9.78(0.58)$ & $9.73(0.60)$ & $9.78(0.62)$ & $9.81(0.54)$ & $9.78(0.55)$ \\
\hline Phosphorus, mg/dL & $3.81(0.56)$ & $3.71(0.57)$ & $3.80(0.56)$ & $3.85(0.56)$ & $3.87(0.56)$ \\
\hline Parathyroid hormone, $\mathrm{pg} / \mathrm{mL}$ & $43(33-57)$ & $41(32-56)$ & $42(33-56)$ & $43(33-56)$ & $47(35-68)$ \\
\hline
\end{tabular}

Values are $\mathrm{n}$, mean $\pm \mathrm{SD}, \mathrm{n}(\%)$, or median (interquartile range).

Abbreviations: FGF23, fibroblast growth factor-23; eGFR, estimated glomerular filtration rate; med., medication; UACR, urine albumin to creatinine ratio.

C-terminal FGF23 remained strongly associated with mortality even after the eGFR and ACR adjustment. Mutual adjustment for ferritin, transferrin saturation, CRP, eGFR, and UACR had similar effects, completely extinguishing the association of intact FGF23 with mortality risk, and only partially attenuating the association of C-terminal FGF23 with mortality. Findings were similar when evaluating $\mathrm{HF}$ as the outcome of interest (see Table 4). Results were also similar when models were further adjusted for demographics and CVD risk factors (Fig. 1). Finally, results appeared similar irrespective of baseline $\mathrm{CKD}$ status $(P$ interactions all $>0.26$; Table 5).

\section{Discussion}

In a well-characterized cohort of community-living older individuals with and without CKD, we assessed the relationship of 2 commonly used FGF23 assays with subsequent risk of mortality, $\mathrm{HF}$, and $\mathrm{MI}$. We demonstrate that, although FGF23 assessed by either assay was strongly associated with mortality and HF risk in unadjusted analysis, the associations were differentially influenced by kidney function, inflammation, and iron deficiency. Most notably, adjustment for kidney function completely extinguished associations of intact FGF23 with mortality and HF risk, whereas kidney function adjustment had much less influence on the association of C-terminal FGF23 with these end points. While markers of iron deficiency and inflammation were more strongly related to C-terminal FGF23 concentrations than with intact FGF23 concentrations in cross-sectional analyses, these parameters were not the main factors responsible for differential associations with clinical end points. All relationships were similar in individuals with and without CKD.

FGF23 is produced as a full-length "intact" molecule that is thought to be the biologically active form 
Table 2. Baseline characteristics by quartiles of C-terminal fibroblast growth factor-23 in community-living older adults

\begin{tabular}{|c|c|c|c|c|c|}
\hline Variable & Full subcohort & Quartile 1 & Quartile 2 & Quartile 3 & Quartile 4 \\
\hline C-terminal FGF23 range, RU/mL & & $<53$ & $53-69$ & $70-95$ & $>95$ \\
\hline No. & 844 & 206 & 205 & 217 & 216 \\
\hline Age, $y$ & $78(5)$ & $78(4)$ & $77(5)$ & $78(5)$ & $79(5)$ \\
\hline Female & $519(62)$ & $110(53)$ & $117(57)$ & $149(59)$ & $143(66)$ \\
\hline Black & $122(15)$ & $41(20)$ & $31(15)$ & $23(11)$ & $27(13)$ \\
\hline$<$ High school education & $131(16)$ & $24(12)$ & $29(14)$ & $38(18)$ & $40(19)$ \\
\hline \multicolumn{6}{|l|}{ Smoking status } \\
\hline Never & $422(51)$ & $106(53)$ & $104(52)$ & $105(49)$ & $107(51)$ \\
\hline Former & $349(42)$ & $87(44)$ & $84(42)$ & $91(43)$ & $87(41)$ \\
\hline Current & $57(7)$ & $7(4)$ & $14(7)$ & $18(8)$ & $18(9)$ \\
\hline Body mass index, $\mathrm{kg} / \mathrm{m}^{2}$ & $27.0(4.8)$ & $26.4(4.2)$ & $26.6(4.3)$ & $26.9(4.7)$ & $28.1(5.6)$ \\
\hline Diabetes & $116(14)$ & $17(8)$ & $25(12)$ & $35(16)$ & $39(18)$ \\
\hline Hypertension & $500(60)$ & $110(53)$ & $116(57)$ & $139(65)$ & $135(63)$ \\
\hline Systolic blood pressure, $\mathrm{mm} \mathrm{Hg}$ & $137(21)$ & $137(21)$ & $136(21)$ & $136(20)$ & $137(22)$ \\
\hline Diastolic blood pressure, $\mathrm{mm} \mathrm{Hg}$ & $69(11)$ & $70(10)$ & $71(10)$ & $69(10)$ & $68(13)$ \\
\hline Heart rate, per min & $63(11)$ & $62(9)$ & $62(10)$ & $63(10)$ & $66(12)$ \\
\hline Antihypertensive med. use & $470(56)$ & $85(41)$ & $109(53)$ & $131(60)$ & $145(67)$ \\
\hline Total cholesterol, mg/dL & $202(39)$ & $200(37)$ & $202(38)$ & $203(38)$ & $201(42)$ \\
\hline Statin use & $96(11)$ & $16(8)$ & $25(12)$ & $27(12)$ & $28(13)$ \\
\hline \multicolumn{6}{|l|}{ Laboratory indices } \\
\hline $\mathrm{eGFR}, \mathrm{mL} / \mathrm{min} / 1.73 \mathrm{~m}^{2}$ & $63(18)$ & $73(15)$ & $68(17)$ & $63(15)$ & $50(18)$ \\
\hline eGFR $<60$ & $354(42)$ & $40(19)$ & $68(33)$ & $92(42)$ & $154(71)$ \\
\hline Urine albumin/creatinine ratio, $\mathrm{mg} / \mathrm{g}$ & $8.60(4.83-19.49)$ & $6.69(4.59-11.81)$ & $8.67(4.78-17.20)$ & $8.37(4.83-18.85)$ & $12.25(5.38-45.73)$ \\
\hline $\mathrm{UACR} \geq 30$ & $148(18)$ & $18(9)$ & $28(14)$ & $37(17)$ & $65(30)$ \\
\hline $25(\mathrm{OH})$ vitamin $\mathrm{D}, \mathrm{ng} / \mathrm{mL}$ & $28(11)$ & $29(10)$ & $29(12)$ & $28(11)$ & $28(12)$ \\
\hline Ferritin, ng/mL & $101(57-176)$ & $127(73-209)$ & $112(63-187)$ & $99(57-175)$ & $70(32-128)$ \\
\hline Transferrin saturation, $\%$ & $30(10)$ & $33(9)$ & $32(9)$ & $31(9)$ & $26(10)$ \\
\hline C-reactive protein, $\mathrm{mg} / \mathrm{L}$ & $2.34(1.04-5.04)$ & $1.72(0.91-3.85)$ & $1.94(0.91-4.19)$ & $2.83(1.24-5.14)$ & $3.22(1.58-7.18)$ \\
\hline Calcium, mg/dL & $9.78(0.58)$ & $9.69(0.57)$ & $9.84(0.60)$ & $9.77(0.57)$ & $9.79(0.61)$ \\
\hline Phosphorus, mg/dL & $3.81(0.56)$ & $3.69(0.57)$ & $3.76(0.53)$ & $3.81(0.59)$ & $3.96(0.53)$ \\
\hline Parathyroid hormone, $\mathrm{pg} / \mathrm{mL}$ & $43(33-57)$ & $40(31-54)$ & $43(31-55)$ & $42(33-57)$ & $49(37-69)$ \\
\hline
\end{tabular}

Values are $\mathrm{n}$, mean $\pm \mathrm{SD}, \mathrm{n}(\%)$, or median (interquartile range).

Abbreviations: FGF23, fibroblast growth factor-23; eGFR, estimated glomerular filtration rate; med., medication; UACR, urine albumin to creatinine ratio.

Table 3. Correlations between fibroblast growth factor-23 assays and iron, inflammatory, and kidney function parameters

\begin{tabular}{|c|c|c|c|c|c|c|c|}
\hline & C-FGF23 & Intact FGF23 & Ferritin & Transferrin sat & CRP & eGFR & UACR \\
\hline C-FGF23 & 1.000 & $0.475^{b}$ & $-0.261^{b}$ & $-0.277^{b}$ & $0.212^{b}$ & $-0.451^{b}$ & $0.194^{b}$ \\
\hline Intact FGF23 & - & 1.000 & 0.037 & -0.032 & $0.087^{a}$ & $-0.419^{b}$ & $0.126^{b}$ \\
\hline Ferritin & - & - & 1.000 & $0.359^{b}$ & 0.043 & 0.011 & 0.018 \\
\hline Transferrin sat & - & - & - & 1.000 & $-0.227^{b}$ & $0.129^{b}$ & -0.025 \\
\hline CRP & - & - & - & - & 1.000 & $-0.143^{b}$ & $0.075^{a}$ \\
\hline eGFR & - & - & - & - & - & 1.000 & $0.199^{b}$ \\
\hline UACR & - & - & - & - & - & - & 1.000 \\
\hline
\end{tabular}

Spearman correlation coefficients are shown for each comparison.

Abbreviations: C-FGF23, C-terminal fibroblast growth factor-23; CRP, C-reactive protein; eGFR, estimated glomerular filtration rate; sat, saturation; UACR, urine albumin to creatinine ratio.

${ }^{a} P$ less than $.05 .{ }^{b} P$ less than .01.

responsible for binding the FGF23 receptor and exerting its biological actions in the kidney. These include induction of phosphaturia and downregulation of $1 \alpha$ hydroxylase, which reduces calcitriol production. Thus, if FGF23 is causally related to clinical end points, then the independent associations of the intact form of FGF23 with clinical end 
Table 4. Impact of iron deficiency, inflammation, and kidney function on the associations of intact and C-terminal fibroblast growth factor-23 (per 2-fold higher) with all-cause mortality, heart failure, and myocardial infarction

\begin{tabular}{|c|c|c|c|c|c|c|c|}
\hline & & & Unadjusted & $\begin{array}{c}\text { Ferritin/Transferrin } \\
\text { sat adjusted }\end{array}$ & CRP adjusted & $\begin{array}{c}\text { eGFR/UACR } \\
\text { adjusted }\end{array}$ & $\begin{array}{l}\text { Mutually } \\
\text { adjusted }^{a}\end{array}$ \\
\hline & Events/N & Rate, \%/y & $\mathrm{HR}(95 \% \mathrm{CI})$ & HR $(95 \% \mathrm{CI})$ & HR (95\% CI) & $\operatorname{HR}(95 \% \mathrm{CI})$ & $\operatorname{HR}(95 \% \mathrm{CI})$ \\
\hline All-cause mortality & $658 / 844$ & 7.81 & & & & & \\
\hline Intact FGF23 & & & $1.45(1.25-1.68)$ & $1.44(1.25-1.67)$ & $1.46(1.25-1.69)$ & $1.00(0.85-1.17)$ & $1.01(0.86-1.19)$ \\
\hline C-FGF23 & & & $1.38(1.26-1.50)$ & $1.52(1.37-1.69)$ & $1.37(1.25-1.50)$ & $1.15(1.04-1.28)$ & $1.24(1.07-1.36)$ \\
\hline Incident heart failure & $220 / 988$ & 3.09 & & & & & \\
\hline Intact FGF23 & & & $1.57(1.25-1.97)$ & $1.55(1.24-1.95)$ & $1.52(1.21-1.91)$ & $0.99(0.76-1.28)$ & $0.97(0.75-1.26)$ \\
\hline C-FGF23 & & & $1.45(1.25-1.68)$ & $1.54(1.29-1.83)$ & 1.39 (1.19-1.62) & $1.16(0.97-1.38)$ & $1.09(0.89-1.34)$ \\
\hline $\begin{array}{l}\text { Incident myocardial } \\
\text { infarction }\end{array}$ & $139 / 878$ & 1.58 & & & & & \\
\hline Intact FGF23 & & & $1.25(0.96-1.65)$ & $1.24(0.95-1.63)$ & $1.23(0.93-1.61)$ & $0.93(0.69-1.24)$ & $0.93(0.69-1.24)$ \\
\hline C-FGF23 & & & $1.17(0.97-1.41)$ & $1.32(1.07-1.63)$ & $1.14(0.94-1.38)$ & $0.96(0.78-1.20)$ & $1.04(0.81-1.32)$ \\
\hline
\end{tabular}

Abbreviations: C-FGF23, C-terminal fibroblast growth factor-23; CRP, C-reactive protein; eGFR, estimated glomerular filtration rate; sat, saturation; HR, hazard ratio; UACR, urine albumin to creatinine ratio.

${ }^{a}$ Simultaneously adjusted for ferritin, transferrin saturation, CRP, UACR, and eGFR.

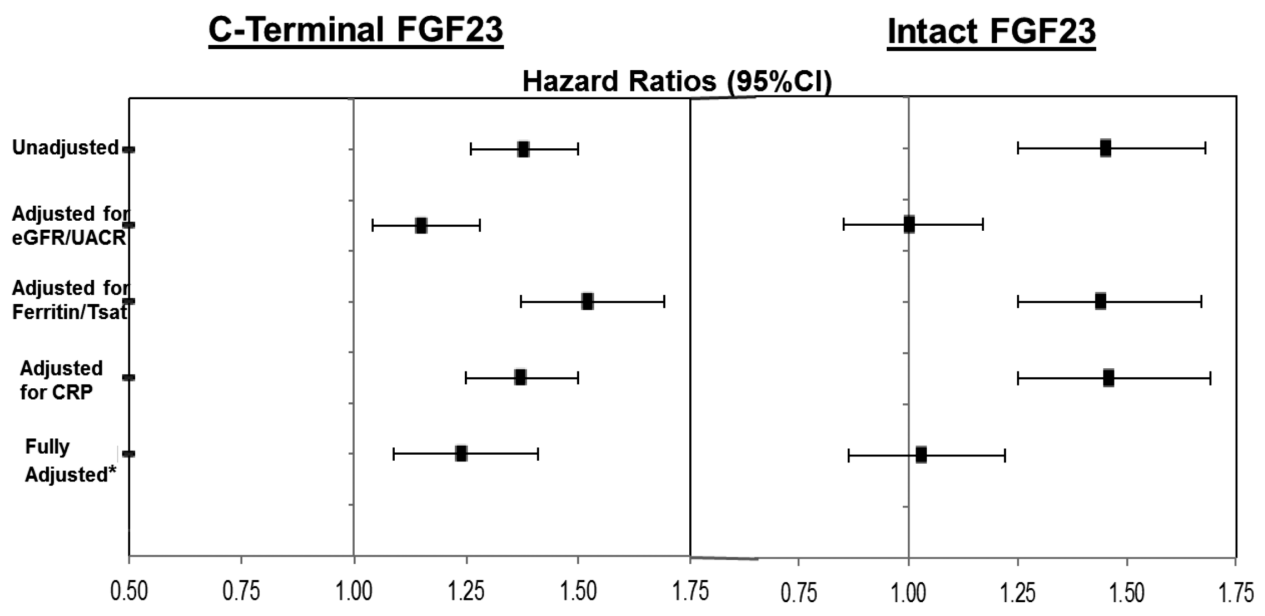

Fully adjusted model includes age, gender, race, education, diabetes, systolic blood pressure, hypertensive meds, BMI, smoking, total cholesterol, calcium, phosphate, parathyroid hormone, 25hydroxy vitamin-D, ferritin, transferrin saturation, c-reactive protein, glomerular filtration rate, and urine albumin creatinine ratio

Figure 1. Effects of adjustment for iron parameters, inflammation, and kidney function on the associations of $\mathrm{C}$-terminal and intact fibroblast growth factor-23 (FGF23) with mortality. Hazard ratios (HRs) represent per 2-fold higher concentrations of FGF23. Fully adjusted model includes age, sex, race, education, diabetes, systolic blood pressure, hypertensive medications, body mass index, smoking, total cholesterol, calcium, phosphate, parathyroid hormone, 25-hydroxyvitamin D, ferritin, transferrin saturation, C-reactive protein (CRP), estimated glomerular filtration rate (eGFR) , and urine albumin to creatinine ratio (UACR).

points should reflect the true strength of such effects. However, much of the enthusiasm for FGF23 as a potential causal intermediary for the adverse outcomes of CKD has been driven by epidemiologic reports of the strong associations of C-terminal FGF23 assay with clinical end points rather than using the intact assay $(19,20)$. This relationship may have been exaggerated in terms of its strength, and overemphasized in terms of the potential impact of FGF23 lowering, if there is indeed no association of intact FGF23 with clinical outcomes after accounting for eGFR and UACR.
Mechanisms explaining why the associations of intact FGF23 with outcomes may be more influenced by eGFR and ACR than associations of C-terminal FGF23 are uncertain. It is possible that the kidneys metabolize FGF23 differently when it is presented as an intact hormone vs as a fragment. Recent studies in humans with acute kidney injury demonstrated detectable FGF23 fragments in urine, also consistent with kidney FGF23 metabolism (21). Studying 17 humans who provided aorta and renal vein specimens, van Ballegooijen and colleagues demonstrated that intact FGF23 concentrations were $17 \%$ lower in the 


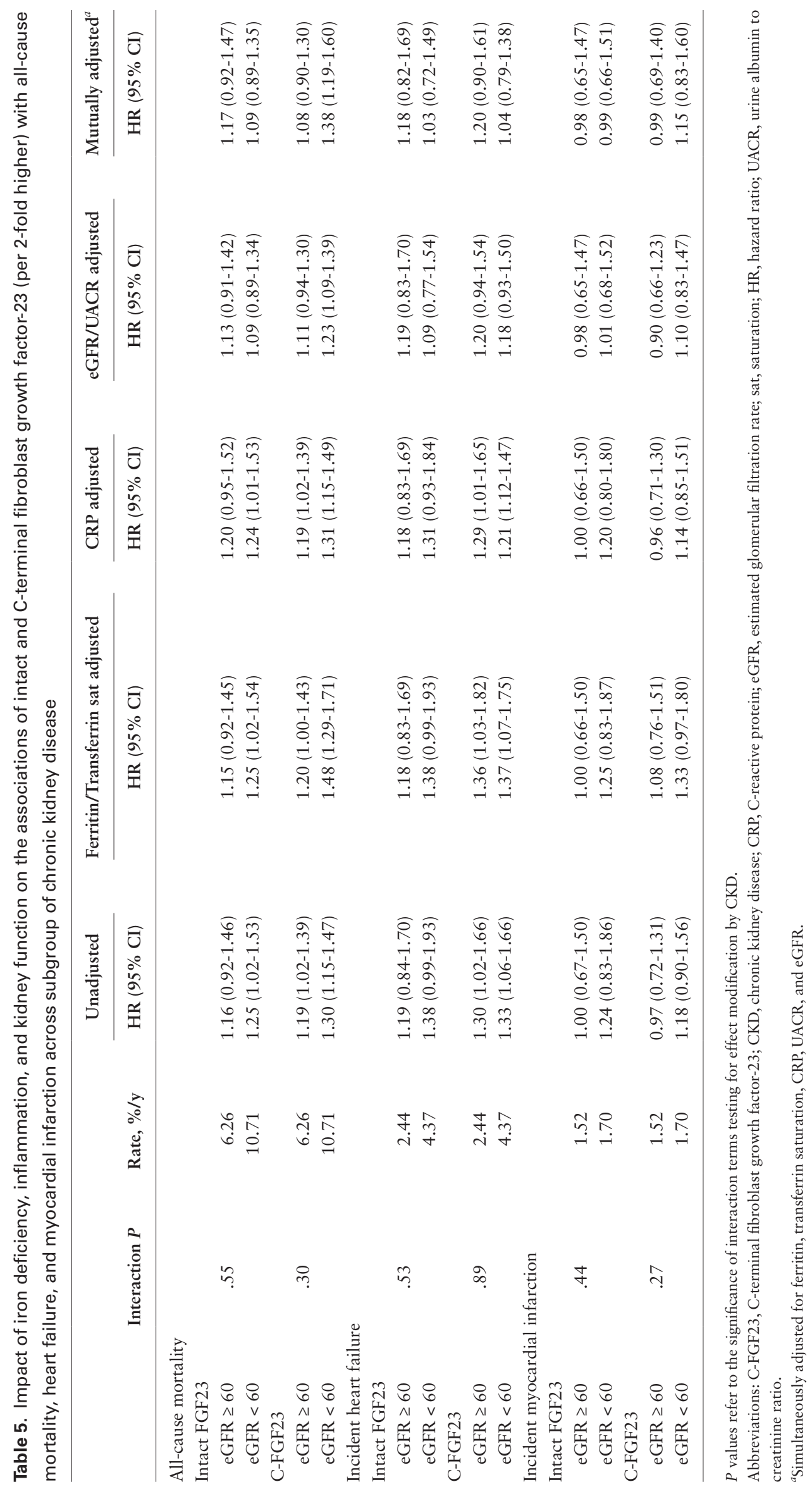


renal vein relative to the aorta. Thus, the kidney appears to be at least partially responsible for clearing intact FGF23 from the circulation (14). However, while the above mentioned studies have evaluated intact FGF23, to our knowledge, no studies have evaluated the effects of kidney clearance on C-terminal FGF23. It is possible that the renal clearance of intact FGF23 is more efficient than C-terminal FGF23, which could account for why there are differential effects of adjustment for kidney function in our study. It seems less likely that differences in measurement techniques may have led to this finding, as the cross-sectional correlations of either FGF23 assay with eGFR and ACR were almost identical in this study, and yet the effects of statistical adjustment for kidney function in longitudinal models was markedly different.

To our knowledge, only one prior study has evaluated the relative associations of C-terminal and intact FGF23 with CVD and HF, and evaluated the attenuation by iron deficiency and kidney function. This study evaluated 575 patients, all with CKD, and provided 5.1 years of follow-up. Similar to our findings, they report that the association of intact FGF23 was attenuated in a multivariable model that included eGFR and ACR along with 7 other risk factors, an association not observed with C-terminal FGF23 (22). However, because all 9 covariates were included simultaneously, it is not possible to dissect whether eGFR, ACR, or other risk factors accounted for the attenuation in this manuscript. In subsequent models, additional adjustment for markers of iron deficiency and inflammation did not appear to meaningfully further attenuate the results. Our findings are consistent, but differ in several important ways. First, we isolate kidney function, iron deficiency, and inflammation in separate models to determine the influence of each of these important covariates, so we can demonstrate specifically that kidney function was the key covariate that attenuated intact FGF23, and did so much more than for C-terminal FGF23. Second, the study population in the prior study all had CKD and were recruited in Germany, thus were likely nearly all Caucasian, although race is not specifically reported. Beyond these factors, our study was much larger, provided many more events, and included people both with and without CKD, providing considerable statistical power to test interactions and allowing us to test and confirm that results are similar in those with and without CKD.

Our findings are also consistent with prior studies demonstrating stronger associations of iron deficiency and inflammation with C-terminal relative to intact FGF23 concentrations. These findings support recent insights into biology influencing FGF23 production and cleavage. Prior studies have demonstrated that iron deficiency, anemia, and hypoxia induce increased production and cleavage of
FGF23, resulting in generally similar intact FGF23 concentrations but elevated C-terminal FGF23 concentrations (12, 13, 23). Similar biological effects are exerted by inflammatory stress. Because inflammation (24), anemia, and iron deficiency (13) are markers of comorbid disease severity, and have themselves been associated with mortality risk, we hypothesized that these factors might account for heterogeneity in the strengths of associations of C-terminal vs intact FGF23 with clinical outcomes. However, accounting for markers of iron deficiency moderately strengthened rather than attenuated the associations of C-terminal FGF23 with mortality and HF risk in this study. Accounting for CRP levels did not materially influence associations by either FGF23 assay type with clinical end points. Thus, our findings suggest that the differential influence of kidney function, rather than iron deficiency or inflammation, appears to be the main factor responsible for differences in association of C-terminal vs intact FGF23 with HF and mortality.

This study has several strengths. We evaluated a wellcharacterized cohort of older community-dwelling adults with long-term follow-up data, a population with high event rates, and nearly half had CKD. Thus, the study had substantial statistical power that provided the opportunity to test our hypotheses with considerable precision, and to evaluate consistency in subgroups with and without CKD. While prior systematic reviews have documented stronger associations in studies that used the C-terminal vs intact FGF23 $(10,11)$, it remained possible that differences in the study populations in the underlying studies, rather than assay type, were responsible for this heterogeneity. The present study is one of few large-scale epidemiologic studies in which both C-terminal and intact FGF23 measurements are available in the same individuals at the same study visit, and demonstrated that C-terminal FGF23 was indeed more strongly associated with these end points within the same individuals. We also directly addressed the influence of iron, inflammation, and kidney function on observed differences in associations with clinical end points within individual participants. Finally, the CHS cohort specifically adjudicated HF and MI events, captured all mortality events, and provided detailed ascertainment of risk factors, covariates, and outcomes.

This study also has important limitations. As with all observational studies, there remains the possibility of residual confounding, and we cannot determine cause-effect relationships of the associations. Our purpose was to root out residual confounding that may have been present in prior studies, and at least for intact FGF23, where the ultimate association reported here was at unity, residual confounding by other covariates seems implausible. We measured both FGF23 assays, markers of iron deficiency, 
and inflammation at one time point, so it remains possible that longitudinal changes in these parameters may differently influence associations with outcomes. Because hemoglobin concentrations were not measured in the CHS, we cannot determine the prevalence of iron deficiency anemia in this cohort. Our study population was limited to elderly Blacks and Whites. Although nearly half of the participants had CKD, few had advanced CKD and the degree of albuminuria was modest. Future studies are required to determine whether the results are generalizable to younger populations, people of other race/ethnicities, or individuals with more advanced CKD.

In conclusion, among community-dwelling older adults, the relationships of FGF23 with clinical end points differ markedly depending on the type of FGF23 assay that is used for measurement. Adjustment for eGFR and ACR completely extinguished the associations of intact FGF23 with mortality and HF; effects only partially observed with C-terminal FGF23. As intact FGF23 measures the biologically active hormone, prior studies reporting strong associations of C-terminal FGF23 with outcomes that are independent of eGFR and ACR may be confounded by kidney function nonetheless. The completely extinguished association of intact FGF23 with clinical end points after adjusting for kidney function suggests that therapies targeting FGF23-lowering may not translate into improved clinical end points.

\section{Acknowledgments}

Financial Support: This work was supported by the National Institute of Diabetes and Digestive and Kidney Diseases (NIDDK, Grants R01DK119528 and K24DK110427 to J.H.I.) The CHS was supported by the National Heart, Lung, and Blood Institute (contracts HHSN268201200036C, HHSN268200800007C, HHSN268201800001C, N01HC55222, N01HC85079, N01HC85080, N01HC85081， N01HC85082， N01HC85083, N01HC85086, and grants U01HL080295 and U01HL130114), the National Institute of Neurological Disorders and Stroke, and the National Institute on Aging (R01AG023629). A full list of principal CHS investigators and institutions can be found at CHS-NHLBI.org. The content is solely the responsibility of the authors and does not necessarily represent the official views of the National Institutes of Health.

Author Contributions: Study design: J.H.I. Data analysis: S.S., R.K., and J.H.I. Drafting of manuscript: S.S. Revising of final version: A.B., P.C., P.W.D., A.A.K., A.H., and M.G.S. Approving final version and decision to submit for publication: J.H.I. J.H.I. had full access to all the data in the study and takes responsibility for the integrity of the data analysis.

\section{Additional Information}

Correspondence and Reprint Requests: Joachim H. Ix, MD, MAS, University of California San Diego, San Diego, CA 92103, USA. E-mail: Joeix@health.ucsd.edu.

Disclosure Summary: The authors have nothing to disclose.
Data Availability: The data sets generated during and/or analyzed during the present study are not publicly available but are available from the corresponding author on reasonable request.

\section{References}

1. Faul C, Amaral AP, Oskouei B, et al. FGF23 induces left ventricular hypertrophy. J Clin Invest. 2011;121(11):4393-4408.

2. Ix JH, Katz R, Kestenbaum BR, et al. Fibroblast growth factor-23 and death, heart failure, and cardiovascular events in community-living individuals: CHS (Cardiovascular Health Study). J Am Coll Cardiol. 2012;60(3):200-207.

3. Lutsey PL, Alonso A, Selvin E, et al. Fibroblast growth factor-23 and incident coronary heart disease, heart failure, and cardiovascular mortality: the Atherosclerosis Risk in Communities study. J Am Heart Assoc. 2014;3(3):e000936.

4. Ix JH, Isakova T, Larive B, et al. Effects of nicotinamide and lanthanum carbonate on serum phosphate and fibroblast growth factor-23 in CKD: the COMBINE trial. J Am Soc Nephrol. 2019;30(6):1096-1108.

5. Lioufas N, Toussaint ND, Pedagogos E, et al; IMPROVE-CKD Writing Committee. Can we IMPROVE cardiovascular outcomes through phosphate lowering in CKD? Rationale and protocol for the IMpact of Phosphate Reduction On Vascular End-points in Chronic Kidney Disease (IMPROVE-CKD) study. BMJ Open. 2019;9(2):e024382.

6. Jonsson KB, Zahradnik R, Larsson $\mathrm{T}$, et al. Fibroblast growth factor 23 in oncogenic osteomalacia and X-linked hypophosphatemia. N Engl J Med. 2003;348(17):1656-1663.

7. Shimada T, Urakawa I, Isakova T, et al. Circulating fibroblast growth factor 23 in patients with end-stage renal disease treated by peritoneal dialysis is intact and biologically active. J Clin Endocrinol Metab. 2010;95(2):578-585.

8. Kestenbaum B, Sachs MC, Hoofnagle AN, et al. Fibroblast growth factor-23 and cardiovascular disease in the general population: the Multi-Ethnic Study of Atherosclerosis. Circ Heart Fail. 2014;7(3):409-417.

9. Ginsberg C, Craven TE, Chonchol MB, et al; SPRINT Research Group. PTH, FGF23, and intensive blood pressure lowering in chronic kidney disease participants in SPRINT. Clin J Am Soc Nephrol. 2018;13(12):1816-1824.

10. Qin Z, Liu X, Song M, et al. Fibroblast growth factor 23 as a predictor of cardiovascular and all-cause mortality in prospective studies. Atherosclerosis. 2017;261:1-11.

11. Xiao Y, Luo X, Huang W, Zhang J, Peng C. FGF 23 and risk of all-cause mortality and cardiovascular events: a meta-analysis of prospective cohort studies. Int J Cardiol. 2014;177(2):575-577.

12. David V, Martin A, Isakova T, et al. Inflammation and functional iron deficiency regulate fibroblast growth factor 23 production. Kidney Int. 2016;89(1):135-146.

13. Kovesdy CP, Estrada W, Ahmadzadeh S, Kalantar-Zadeh K. Association of markers of iron stores with outcomes in patients with nondialysis-dependent chronic kidney disease. Clin J Am Soc Nephrol. 2009;4(2):435-441.

14. van Ballegooijen AJ, Rhee EP, Elmariah S, de Boer IH, Kestenbaum B. Renal Clearance of mineral metabolism biomarkers. J Am Soc Nephrol. 2016;27(2):392-397.

15. Smith ER, Cai MM, McMahon LP, Holt SG. Biological variability of plasma intact and C-terminal FGF23 measurements. $J$ Clin Endocrinol Metab. 2012;97(9):3357-3365. 
16. Inker LA, Schmid $\mathrm{CH}$, Tighiouart $\mathrm{H}$, et al; CKD-EPI Investigators. Estimating glomerular filtration rate from serum creatinine and cystatin C. N Engl J Med. 2012;367(1):20-29.

17. Wacholder S, Boivin JF. External comparisons with the casecohort design. Am J Epidemiol. 1987;126(6):1198-1209.

18. Barlow WE. Robust variance estimation for the case-cohort design. Biometrics. 1994;50(4):1064-1072.

19. Isakova T, Xie H, Yang W, et al; Chronic Renal Insufficiency Cohort (CRIC) Study Group. Fibroblast growth factor 23 and risks of mortality and end-stage renal disease in patients with chronic kidney disease. JAMA. 2011;305(23):2432-2439.

20. Gutiérrez OM, Mannstadt M, Isakova T, et al. Fibroblast growth factor 23 and mortality among patients undergoing hemodialysis. N Engl J Med. 2008;359(6):584-592.
21. Leaf DE, Jacob KA, Srivastava A, et al. Fibroblast growth factor 23 levels associate with AKI and death in critical illness. J Am Soc Nephrol. 2017;28(6):1877-1885.

22. Emrich IE, Brandenburg V, Sellier AB, et al. Strength of fibroblast growth factor 23 as a cardiovascular risk predictor in chronic kidney disease weaken by ProBNP adjustment. Am J Nephrol. 2019;49(3):203-211.

23. Clinkenbeard EL, Hanudel MR, Stayrook KR, et al. Erythropoietin stimulates murine and human fibroblast growth factor-23, revealing novel roles for bone and bone marrow. Haematologica. 2017;102(11):e427-e430.

24. Reuben DB, Cheh AI, Harris TB, et al. Peripheral blood markers of inflammation predict mortality and functional decline in high-functioning community-dwelling older persons. J Am Geriatr Soc. 2002;50(4):638-644. 